G589(P) CHARACTERISATION OF PAEDIATRIC SAFETY INCIDENTS IN GENERAL PRACTICE: A MIXED METHODS ANALYSIS

${ }^{1} \mathrm{P}$ Rees, ${ }^{1} \mathrm{~A}$ Edwards, ${ }^{2} \mathrm{~S}$ Panesar, ${ }^{3} \mathrm{C}$ Powell, ${ }^{1} \mathrm{~B}$ Carter, ${ }^{1} \mathrm{H}$ Williams, ${ }^{4} \mathrm{P}$ Hibbert, ${ }^{5} \mathrm{D}$ Luff, ${ }^{6} \mathrm{G}$ Parry, ${ }^{1} \mathrm{~S}$ Mayor, ${ }^{7} \mathrm{~A}$ Avery, ${ }^{2} \mathrm{~A}$ Sheikh, ${ }^{8} \mathrm{~L}$ Donaldson, ${ }^{1} \mathrm{~A}$ Carson-Stevens. ${ }^{1}$ Cochrane Institute for Primary Care and Public Health, Cardiff University, Cardiff, UK; ${ }^{2}$ Centre for Population Health Sciences, University of Edinburgh, Edinburgh, UK; ${ }^{3}$ Institute of Molecular and Experimental Medicine, School of Medicine, Cardiff University, Cardiff, UK; ${ }^{4}$ Australian Institute for Healthcare Innovation, University of New South Wales, Australia; ${ }^{5}$ Institute for Professionalism and Ethical Practice/Department of Anesthesia Boston Children's Hospital, USA; ' Institute for Healthcare Improvement, Massachusetts, Boston, USA; 'Faculty of Medicine and Health Sciences, University of Nottingham, Nottingham, UK; ${ }^{8}$ Department of Surgery \& Cancer, Imperial College London, London, UK

\subsection{6/archdischild-2015-308599.538}

Aim Healthcare failures have been identified in $26 \%$ of UK child deaths. Despite children accounting for $40 \%$ of general practitioners' workload, little is known about the safety of care in the community setting. Using data from a national reporting system, this study aimed to characterise the paediatric safety incidents occurring in general practice to inform a logic model with identified priorities for clinical practice improvement.

Methods We undertook a retrospective cross-sectional mixed methods study utilising paediatric reports submitted to the National Reporting and Learning System from general practice (2003-2013). Analysis involved a detailed data coding process using multi-axial frameworks combined with descriptive statistical analysis, and thematic analysis of all reports of severe harm and death. A 20\% sample of reports were independantly double coded and kappa statistics of inter-rater reliability were calculated. Using frequency distributions and cross-tabulations the relationships between incident types and contributory factors were explored. Clusters of contributory factors were identified. Subject matter experts identified primary and secondary drivers for improvement.
Results 1,788 reports were identified with 763 (42.7\%) describing harm to children. Four priority areas-incidents associated with the most harmful outcomes-include errors associated with: medication management; timely referral of unwell children; delivery of safe evidence-based treatment; and, adequate diagnosis and assessment. Poor referral and treatment decisions in severely unwell or vulnerable children, along with delayed diagnosis and insufficient assessment of such children, featured prominently in incidents resulting in severe harm or death. The factors contributing to these incidents form the bases of our recommendations for improvement and are illustrated in a logic model (see Figure 1).

Conclusion This is the first analysis of nationally-collected general practice-related paediatric safety incident reports. Recommendations to mitigate harm in these priority areas include: mandatory paediatric training for all general practitioners; utilising electronic tools to support diagnosis, management and referral decision-making; and use of tools such as bar code scanning to reduce medication errors. Practitioners are encouraged to reflect on these recommendations, as well as explore their own local safety report data to identify additional priorities for practice improvement.

\section{G590(P) INTRODUCING DELAYED CORD CLAMPING AS ROUTINE PRACTICE IN A TERTIARY NEONATAL UNIT}

SC Jaques. Neonatal Unit, St George's Hospital, London, UK

\subsection{6/archdischild-2015-308599.539}

Background Delaying cord clamping (DCC) following delivery of an infant allows for placental transfusion and transfer of blood from the placenta to the infant. Up to two thirds of an infant's circulating volume will be within the placenta at delivery. In premature babies delaying clamping of the umbilical cord has

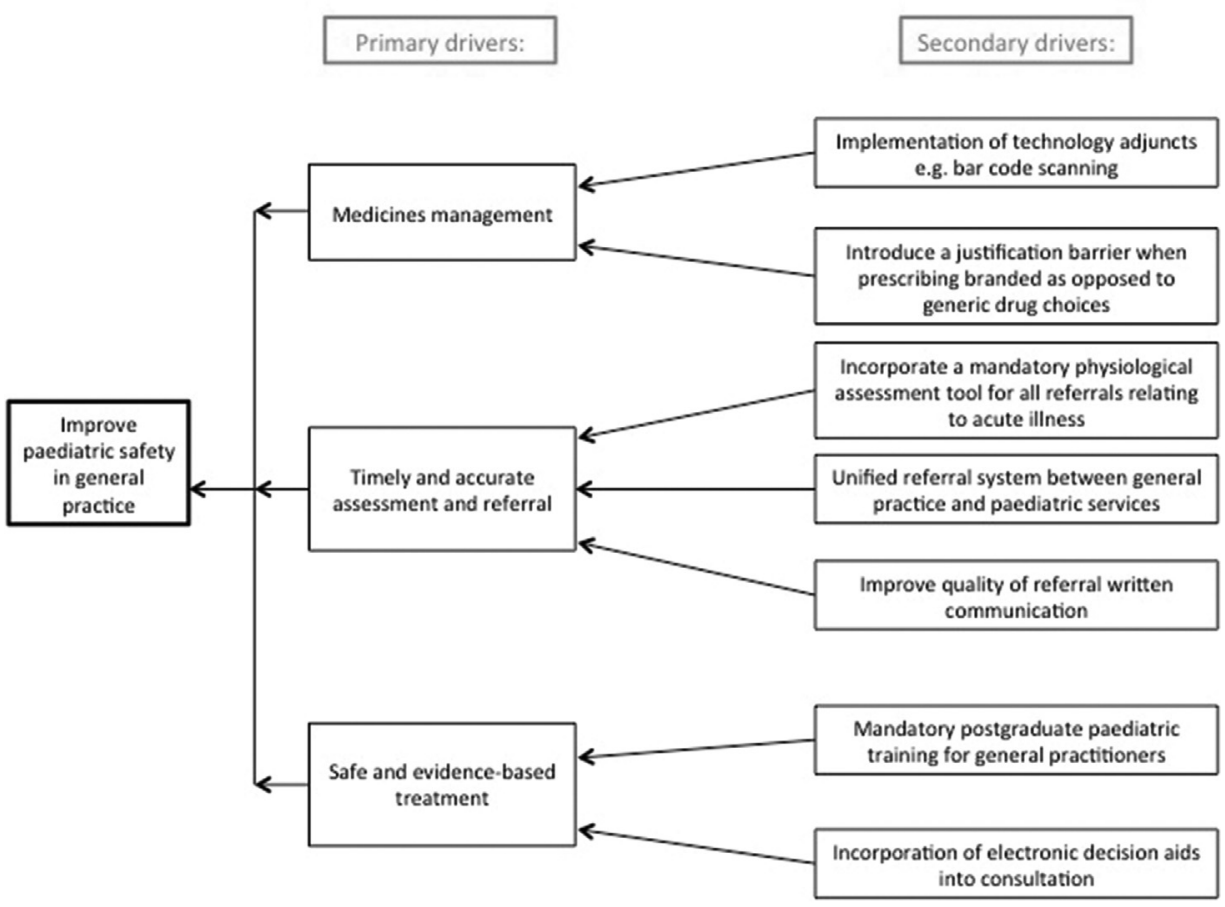

Abstract G589(P) Figure 1 